changed the recommended dosing from $6.5 \mathrm{mg} / \mathrm{kg}$ to $5 \mathrm{mg} /$ $\mathrm{kg}$. However, it is not clear that the lower dose of hydroxychloroquine will have the same efficacy for SLE activity or the same protective role against cardiovascular risk factors and thrombosis. We asked whether hydroxychloroquine blood levels could help identify those at greater future risk of retinopathy.

Methods We analyzed data on 537 SLE patients from a clinical cohort who had repeated assessments of HCQ blood concentrations, and were evaluated one or more times for retinopathy (300 single retinopathy exam, 149 two and 88 three or more assessments). The patients were 92\% female and 42\% Caucasian. Hydroxychloroquine blood levels were performed as previously described. In our analysis, HCQ toxicity was defined dichotomously by a retina expert: all those with a value of No or Possible were categorized as not having HCQ toxicity, and those who had a Yes were categorized as having it. Mean and maximum HCQ blood concentration over all cohort visits prior to the final retinopathy assessment were calculated. Risk of HCQ toxicity was then assessed in tertiles defined by these variables.

Results Significant risk factors for retinal toxicity are shown in table 1.

Conclusions Our data show that the risk of HCQ retinopathy is higher in men and Caucasians. As expected, it is higher in older patients and with greater duration. We also found that BMI and hypertension were predictive of HCQ retinopathy. For the first time, our data show the utility of HCQ blood levels in predicting retinopathy. This would allow clinicians to either decrease dose or increase monitoring in those with high blood levels.

Funding Source(s): The Hopkins Lupus Cohort was funded by NIH Grant R01-AR069572.

\section{TYPE I INTERFERON MODULATES ADAM17 ACTIVITY IN PHOTOSENSITIVE LUPUS MOUSE MODELS}

${ }^{1}$ Thomas M Li*, ${ }^{1}$ Noa Shwartz, ${ }^{1}$ William D Shipman, ${ }^{2}$ Theresa T Lu. ${ }^{1}$ Hospital for Special Surgery; ${ }^{2}$ Autoimmunity and Inflammation Program and Pediatric Rheumatology, Hospital for Special Surgery

\subsection{6/lupus-2019-Ism.17}

Background Systemic lupus erythematosus (SLE) is an autoimmune disease characterized by systemic disease flares, renal complications, photosensitivity, and other clinical manifestations. A research area of particular interest to our lab is photosensitivity, an immune system reaction precipitated by ultraviolet radiation (UVR) exposure that results in epidermal keratinocyte apoptosis and contributes to overall skin inflammation. Importantly, many SLE patients attribute a decrease in quality of life due to photosensitivity. Our lab has recently identified a mechanism in which a disintegrin and metalloprotease 17 (ADAM17) located on Langerhans cells (LCs) limits UVR-induced keratinocyte apoptosis and skin injury via keratinocyte epidermal growth factor receptor (EGFR) stimulation in mice and humans. While we have delineated the downstream effects of UVR-induced ADAM17 activity and expression in healthy and SLE skin, factors that regulate ADAM17 remain poorly understood. Previous studies have shown that elevated levels of type I interferon (IFN) are characteristic of SLE patients. Here, we hypothesize that type I interferon may regulate ADAM17 in LCs in lupus models.

Methods To quantify ADAM17 activity, we developed an assay that uses flow cytometry and co-culture systems. We use this assay in in vitro models and in vivo mouse models. To quantify the relative ratio of IFN-inducible genes, we use quantitative polymerase chain reaction (qPCR). To apply IFN-k to mice, we solubilize the protein and paint it on murine back skin.

Results In vitro methods showed that type I IFN was sufficient to reduce LC ADAM17 activity, and in vivo models showed that type I IFN receptor blockade corrected the LC ADAM17 defect in photosensitive lupus mouse models. We are analyzing qPCR data.

Conclusions We show that type I interferon (IFN) can reduce ADAM17 activity on epidermal cells. We also show that type I interferon receptor blockade in lupus mouse models rescues ADAM17 activity. Our data together suggest that type I IFN in lupus may contribute to Langerhans cell dysfunction and propensity to photosensitivity in SLE.

Funding Source(s): None

\section{AN UNCOMMON OVERLAP OF TWO COMMON RHEUMATOLOGICAL DISORDERS}

Sandesh Guleria*, Ankur Jindal, Sanjeev Naganur, Deepti Suri, Surjit Singh. Postgraduate Institute of Medical Education and Research, Chandigarh, India

\subsection{6/lupus-2019-Ism.18}

Background Juvenile systemic lupus erythematosus (SLE) is a heterogeneous multisystem autoimmune disease. Kawasaki disease $(\mathrm{KD})$ is a common vasculitic disorder in children that manifests with fever and mucocutaneous involvement. While overlap of childhood SLE with other rheumatologic disorders has been described, it is extremely unusual in the
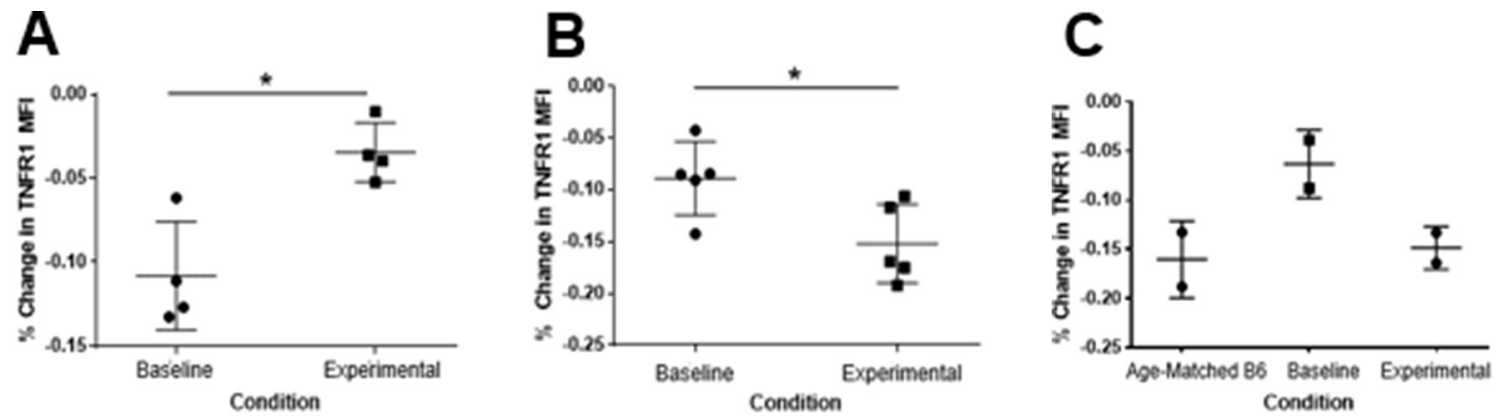

Abstract 17 Figure 1 Interferon is a sufficient and necessary condition to downregulate ADAM17 activity 


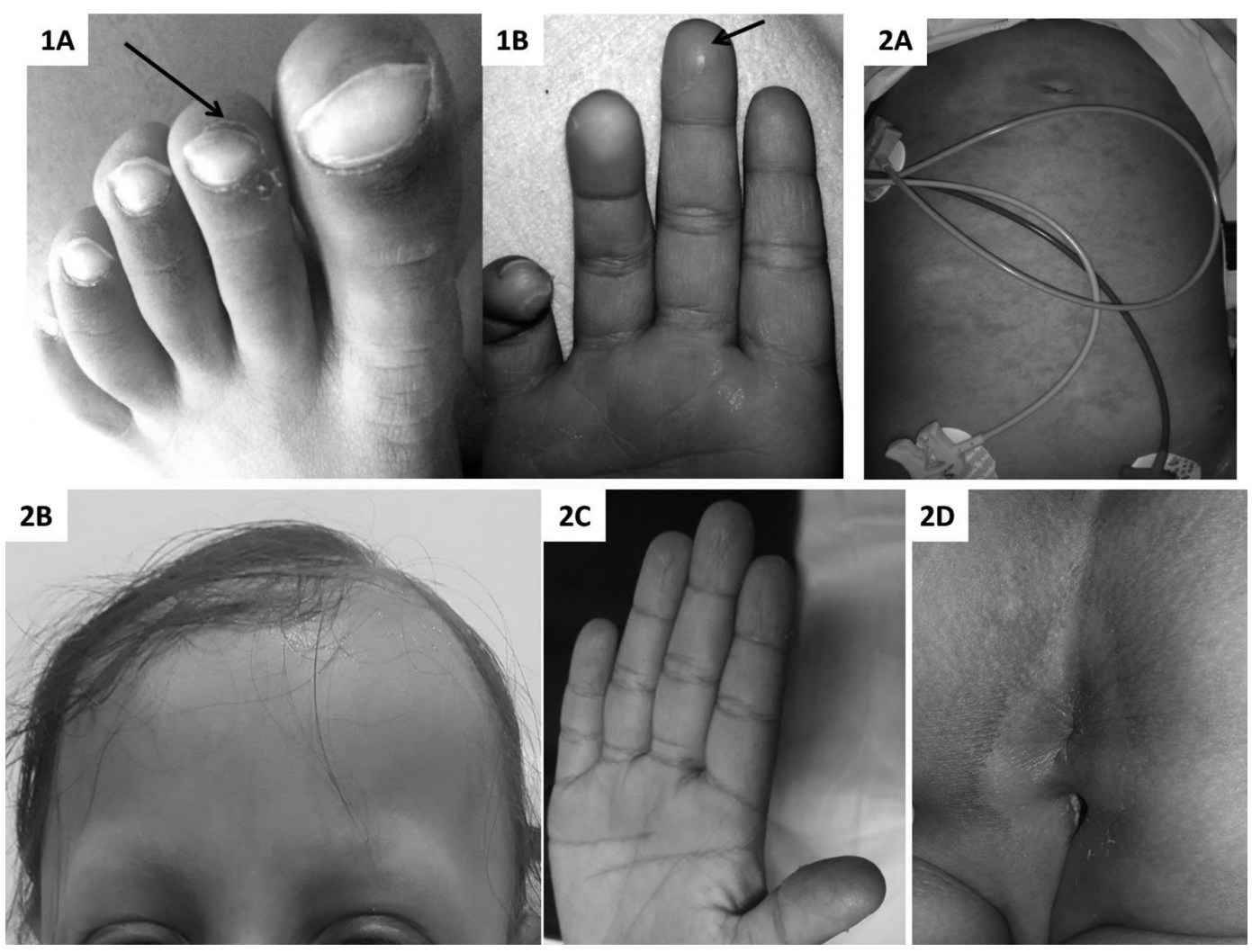

Abstract 18 Figure 1 (1A) Periungual skin peeling in the 2nd toe of left foot and the middle finger of the right hand (1B) in first child; (2A) Erythematous maculopapular rash on trunk in second child; (2B) diffuse alopecia with frontal baldness in second child; (2C) periungual skin peeling in all digits of right hand of second child; (2D) characteristic perianal skin peeling in second child.

context of $\mathrm{KD}$. We report 2 children who concomitantly had SLE and KD.

Methods Patient 1: A 5-year-old boy presented with fever of 2 months. He was irritable, he had malar rash, generalized erythematosus maculopapular rash, oral ulcers, red cracked lips, conjunctival injection, cervical adenopathy and hepatomegaly. Investigations showed anemia, leucopenia, elevated erythrocyte sedimentation rate (ESR) and high C-reactive protein (CRP). He had hypocomplimentemia; positive antinuclear antibody (ANA) (4+diffuse pattern) and high antidsDNA antibody titre $(1560 \mathrm{IU} / \mathrm{mL})$. Diagnosis of SLE was made. One week later he was brought with typical periungual peeling of skin in fingers and toes (figure 1). Investigations showed anemia (haemoglobin- $83 \mathrm{gm} / \mathrm{L}$ ), thrombocytosis $(510 \times 109 / \mathrm{L})$, persistently elevated ESR $(61 \mathrm{~mm}$ in 1 st hour) and high CRP $(16 \mathrm{mg} / \mathrm{L})$. So diagnosis of concomitant $\mathrm{KD}$ was considered.

Patient 2: A 8 year-old-girl presented with diffuse non scarring alopecia for 2 years and fever for 3 weeks. On examination, she had pallor; red cracked lips; palmar erythema; diffuse alopecia (figure1); generalized lymphadenopathy; hepatomegaly. Investigations revealed anaemia, lymphocytopenia, elevated ESR and CRP, high serum pro-BNP levels. She had hypocomplimentemia; positive ANA (4+diffuse pattern) and positive anti-dsDNA antibody. A diagnosis of SLE was made. But, on day 5 of hospital stay she developed periungual and perianal peeling (figure 1), and chromonychia. On reviewing history, she also had redness of lips and tongue, conjunctival injection and generalized maculopapular rash 5 days prior to admission. Possibility of concomitant KD was considered.

Results Both children fulfilled the classification criteria for diagnosis of SLE and KD as well and time course of events was also very suggestive. Patient 1 was administered intravenous immunoglobulin (IVIG) ( $\mathrm{gm} / \mathrm{kg})$, following which his irritability subsided. Patient 2 was also given IVIG $(2 \mathrm{gm} / \mathrm{kg})$. Both patients were discharged on oral prednisolone, hydroxychloroquine and low dose aspirin. Aspirin was stopped after 6 weeks as echocardiography was normal. Both continue to remain well on follow-up.

Conclusions While overlap of childhood SLE with other rheumatological disorders has been described, concomitant occurrence of SLE and $\mathrm{KD}$ is an extremely rare. Two or more rheumatologic diseases may coexist at the same time and one must always be vigilant.

Funding Source(s): No funding source.

\section{EVALUATING MEANINGFUL CHANGE IN DISEASE ACTIVITY AS A CLINICAL EFFICACY MEASURE FOR CLINICAL TRIALS IN CUTANEOUS LUPUS ERYTHEMATOSUS}

${ }^{1}$ Srita Chakka*, 'Rebecca L Krain, ${ }^{1}$ Sarah Ahmed, ${ }^{1}$ Rui Feng, ${ }^{2}$ Victoria P Werth. 'University of Pennsylvania; ${ }^{2}$ Perelman School of Medicine, University of Pennsylvania

\subsection{6/lupus-2019-|sm.19}

Background To date, there are no approved treatments for cutaneous lupus erythematous (CLE), a disease known to significantly burden a patients quality of life (QoL). Clinical trials are important for the advancement of treatments for CLE and trial outcome measures should reflect clinically meaningful improvement in disease activity and its effect on QoL. Currently, trials use an efficacy measure of 50\% improvement in 